\title{
YKL-40: An Emerging Biomarker of Endothelial Dysfunction in Arteriogenic Erectile Dysfunction
}

\section{Original Article}

\author{
Hanan H. Sabry', Ahmed H. Hamed ${ }^{I}$, Jehan H. Sabry'2, Osama H. Abdel-Salama ${ }^{1}$ \\ ${ }^{I}$ Departments of Dermatology Venereology and Andrology, ${ }^{2}$ Clinical and chemical Pathology, \\ Faculty of Medicine, Benha University, Benha, Egypt
}

\begin{abstract}
Introduction: Endothelial dysfunction is a central etiologic factor in the development of atherosclerosis and systemic vascular disease, which includes erectile dysfunction.

YKL-40 has been suggested to be a new marker of inflammation, atherosclerosis, and endothelial dysfunction.

Aim: To estimate serum levels of YKL40, as a new serum marker of endothelial dysfunction, in patients with arteriogenic erectile dysfunction.

Patients and Methods: Hundred subjects including 50 with arteriogenic erectile dysfunction and 50 healthy as a control group were enrolled to the study. Serum YKL-40 levels were measured in patients and controls using ELISA technique. Results: Serum YKL-40 levels was significantly elevated in arteriogenic ED patients compared with controls. Positive significant correlations were found between serum levels of YKL40 and patients' age $(\mathrm{r}=0.588, p=0.001)$, duration of erectile dysfunction $(\mathrm{r}=0.673, p=0.001)$, BMI $(\mathrm{r}=0.598, p=0.001)$. The patients with hypertension had significantly elevated YKL-40 levels than those who were normotensive (164.88 \pm 191.73 Vs $60.22 \pm 26.44$, respectively).

Conclusion: Serum levels of YKL-40 are elevated in arteriogenic ED patients denoting that endothelial dysfunction play a role in the pathogenesis of arteriogenic ED and YKL-40 as a novel marker of endothelial dysfunction could be a marker of arteriogenic erectile dysfunction.
\end{abstract}

Key Words: Arteriogenic, ED, endothelial dysfunction, YKL-40.

Revised: 11 April 2017, Accepted: 9 September 2017

Corresponding Author: Hanan H. Sabry, Tel.: 0133212990, E-mail: hanansabry20032003@yahoo.com

ISSN: 2090-6048, September 2017, Vol. 7, No. 3

\section{INTRODUCTION}

Erectile dysfunction (ED) is defined as the inability to attain or maintain an erection sufficient for satisfactory sexual performance. The nature of men's erections diminishes gradually over time. Therefore, men may have doubts whether their erectile troubles are lasting or brief. The distress of having erectile dysfunction symptoms may prompt to denial of the problem ${ }^{[1]}$.

Cardiovascular disease and erectile dysfunction have several prevalent risk factors with for example, obesity, metabolic syndrome, and smoking, lack of exercise, diabetes, and hypercholesterolemia. Erectile dysfunction was more common in men with a body mass index (BMI) of 30 or more ${ }^{[2]}$.

Erectile dysfunction, aging, and endothelial dysfunction are closely related to each other. Minor risk factors such as inflammation, hypoxia, oxidative stress, and hyperhomocysteinemia are additionally related to ED and endothelial dysfunction. Organic ED comprise up to $80 \%$ of cases, while vascular disease is the most common pathophysiology of $\mathrm{ED}^{[3]}$.

In the presence or absence of cardiovascular risk factors, ED might be an early manifestation of endothelial dysfunction. Therefore, men with ED might be at increased risk for cardiovascular risks and ED might be considered as a sentinel symptom in patients with mysterious cardiovascular disease $(\mathrm{CVD})^{[4]}$.

YKL-40 is a chitinase-like protein. It is expressed by different cell types, including neutrophils and macrophages, while macrophages have been recognized as its main cellular source. Monocytes do not express YKL-40, and YKL-40 expression appears to be associated with later stages of macrophage differentiation. Although 
its biological function is largely unknown, YKL-40 has been suggested to have a role in a variety of processes, including epithelial-mesenchymal transition, migration and proliferation of malignant cells, angiogenesis, tissue remodeling and inflammation ${ }^{[5]}$.

The knowledge of YKL-40 as an early marker of endothelial dysfunction indicates that YKL-40 could possibly correlate with other early markers of endothelial activation and/or dysfunction. YKL-40 levels were found to be higher in individuals with macrovascular complications $^{[6]}$.

\section{AIM}

The aim of the present study was to estimate the levels of YKL40 as a serum marker of endothelial dysfunction in patient with arteriogenic erectile dysfunction.

\section{PATIENTS AND METHODS}

This case-control study included 50 male patients aged between 2550- years old with arteriogenic erectile dysfunction (the diagnosis was confirmed by penile duplex ultrasonography), and 50 healthy age and BMI matched males as a control group not complaining of ED.

They were recruited from the Outpatient Clinic of Dermatology and Andrology of Benha University Hospital in the period from March 2015 to March 2016. All the participants provided informed consents to participate in this study. The laboratory investigations were carried out at the Clinical Pathology Department of Benha University Hospital under complete aseptic conditions. This study was conducted in accordance with the principles of the Declaration of Helsinki and Good Clinical Practices. The study was approved by the Research Ethics Committee of Benha Faculty of Medicine, Benha University, Egypt on January 2015.

Patients with psychogenic erectile dysfunction and other causes of organic ED or those $>50$ years old were excluded from the study.

\section{Methods}

\section{History taking and clinical examination}

Full medical history taken from the patients stressing on, age, onset, course and duration of ED, lifestyle behaviors (smoking), history of drug intake, systemic diseases, and any surgical operations.

General and local examinations: stressing on other causes of erectile dysfunction.

Clinical anthropometry including: weight, height and BMI $(\mathrm{Kg} / \mathrm{m} 2)$.

\section{Laboratory investigations:}

Blood sampling: $5 \mathrm{mls}$ venous blood samples were withdrawn from each patient and control subject using serum separator tubes. Samples were allowed to clot for 30 mins at room temperature, then were centrifugated for 15 mins at $1000 \mathrm{Xg}$. Serum was separated, aliquoted and stored at $-20 \mathrm{C}^{\circ}$. Serum of patients was used for measuring YKL40, cholesterol, triglycerides, and HDL-C. Serum YKL40 levels was measured using ELISA kits which was provided by WKEA MED SUPPLIES CORP, Changchun 130012, China, according to the manufacurer's instructions. Briefly this assay employs the quantitative sandwich enzyme immunoassay technique. A monoclonal antibody specific for human YKL-40 has been pre-coated onto a microplate. Standards and samples were pipetted into the wells and any YKL-40 present was bound by the immobilized antibody. After washing away any unbound substances, an enzymelinked polyclonal antibody specific for human YKL-40 was added to the wells. Following a wash to remove any unbound antibody-enzyme reagent, a substance solution was added to the wells and color develops in proportion to the amount of YKL-40 bound in the initial step. The color development was stopped and the intensity of the color was measured ${ }^{[7]}$.

\section{Statistical Analyses}

The data collected were tabulated and analyzed using the Statistical Program for Social Science (version 20; SPSS Inc., Chicago, Illinois, USA). Categorical data were presented as number and percentages, whereas quantitative data were expressed as mean \pm SD and range. The continuous variables between 2 groups were compared with Student $t$ test. The categorical data were assessed through Pearson's chi-square test. Whereas $\chi 2$-test was used to compare frequencies. Pearson correlation was used to determine relationships. $P$ value $<0.05$ was considered statistically significant.

\section{RESULTS}

The study population consisted of 50 arteriogenic ED patients (mean age 36.06 \pm 6.75 years) and 50 controls (mean age $37.76 \pm 5.97$ years). The age and BMI distributions did not differ significantly between the groups. There was no significant difference between patients and controls as regards smoking, alcohol, and diabetes, The incidence of hypertension was significantly higher in ED group 39 (78\%) patients as compared to controls $21(42 \%) ; p=0.001$.

The clinical and laboratory characteristics of arteriogenic ED patients and the control subjects are presented in Table 1.

Serum YKL40 levels was significantly higher in ED patients than controls $(141.85 \pm 174.84$ vs $62.94 \pm$ 
43.34; $p=0.003)$. The difference between ED patients and controls in lipid profile was insignificant $(p>0.05)$.

The mean serum levels of YKL- 40 in hypertensive ED patients $(164.88 \pm 191.73 \mathrm{ng} / \mathrm{mL})$ was significantly higher $(P<0.05)$ than that measured in serum samples of non-hypertensive patients $(60.22 \pm 26.44 \mathrm{ng} / \mathrm{mL})$. No significant differences were observed in serum YKL- 40 levels measured amongst diabetics' vs. non diabetics or smokers'vs. nonsmokers ED patients Table 2.
Serum levels of YKL40 correlated positively with age of patients $(\mathrm{r}=0.588 ; p<0.001)$, duration of erectile dysfunction $(\mathrm{r}=0.673 ; p<0.001)$, and BMI $(\mathrm{r}=0.598$; $p<0.001$ ) (Fig $1-3$ ).

ROC curve of YKL-40 levels were shown in Fig.4. The optimum cut-off value of YKL-40 concentration was $50 \mathrm{ng} / \mathrm{ml}$ for distinguishing arteriogenic ED patients from healthy controls, with $85 \%$ sensitivity, $68 \%$ specificity and $73 \%$ accuracy.

Table 1: Demographics and Laboratory characteristics of ED patients and controls

\begin{tabular}{|c|c|c|c|c|c|}
\hline \multicolumn{2}{|c|}{ Variable } & Patients $(n=50)$ & Controls $(\mathrm{n}=50)$ & test & $p$. value \\
\hline \multicolumn{2}{|c|}{${ }^{a}$ Age (years) } & $36.06 \pm 6.75$ & $37.76 \pm 5.97$ & t. 1.780 & 0.185 \\
\hline \multicolumn{2}{|c|}{${ }^{\mathrm{a}} \mathrm{BMI}\left(\mathrm{Kg} / \mathrm{m}^{2}\right)$} & $29.30 \pm 2.58$ & $28.41 \pm 2.59$ & t. 2.681 & 0.112 \\
\hline \multicolumn{2}{|c|}{${ }^{a}$ Duration (years) } & $4.8 \pm 3.32$ & & & \\
\hline \multicolumn{2}{|c|}{${ }^{\mathrm{b}}$ Smoking No. (\%) } & $40(80.0 \%)$ & $36(72.0 \%)$ & $\mathrm{X} 2=0.884$ & 0.349 \\
\hline${ }^{b} \mathrm{DM}$ & No. (\%) & $32(64.0 \%)$ & $24(36.0 \%)$ & $X 2=2.603$ & 0.107 \\
\hline${ }^{\mathrm{b}} \mathrm{HTN}$ & No. (\%) & $39(78.0 \%)$ & $30(60.0 \%)$ & $\mathrm{X} 2=3.786$ & 0.052 \\
\hline${ }^{\mathrm{a}} \mathrm{CH}$ & $\mathrm{mg} / \mathrm{dL}$ & $169.80 \pm 41.25$ & $167.12 \pm 36.16$ & t. 0.119 & 0.730 \\
\hline${ }^{\mathrm{a}} \mathrm{TG}$ & $\mathrm{mg} / \mathrm{dL}$ & $147.64 \pm 31.14$ & $140.04 \pm 26.74$ & t. 1.714 & 0.194 \\
\hline${ }^{\mathrm{a}} \mathrm{HDL}$ & $\mathrm{mg} / \mathrm{dL}$ & $67.36 \pm 16.86$ & $69.80 \pm 12.75$ & t. 0.666 & 0.416 \\
\hline${ }^{a} \mathrm{LDL}$ & $\mathrm{mg} / \mathrm{dL}$ & $85.28 \pm 34.70$ & $76.32 \pm 30.99$ & t. 1.855 & 0.176 \\
\hline${ }^{\mathrm{a}} \mathrm{YKL}$ & $(\mathrm{ng} / \mathrm{ml})$ & $141.85 \pm 174.84$ & $62.94 \pm 43.34$ & t. 9.595 & $0.003^{*}$ \\
\hline
\end{tabular}

${ }^{\mathrm{a}} \mathrm{Mean} \pm \mathrm{SD} \quad{ }^{\mathrm{b}}$ No. $(\%)$

Significant $(P<0.05)$

Table 2: Effect of smoking, diabetes and hypertension on serum YKL-40 levels in ED patients

\begin{tabular}{|c|c|c|c|c|c|}
\hline YKL 40 level & & Range & Mean \pm S. D & t. test & p. value \\
\hline \multirow{3}{*}{ Smoking } & Yes & $34.8-850.6$ & $159.81 \pm 191.26$ & \multirow{3}{*}{2.160} & \multirow{3}{*}{0.148} \\
\hline & & & & & \\
\hline & No & $25.2-101.3$ & $70.01 \pm 27.49$ & & \\
\hline \multirow{3}{*}{ DM } & Yes & $25.2-850.6$ & $141.85 \pm 174.84$ & \multirow{3}{*}{0.397} & \multirow{3}{*}{0.532} \\
\hline & & & & & \\
\hline & No & $34.8-310.6$ & $120.96 \pm 80.02$ & & \\
\hline \multirow{3}{*}{ HTN } & Yes & $40.8-850.6$ & $164.88 \pm 191.73$ & \multirow{3}{*}{4.213} & \multirow{3}{*}{$0.032 *$} \\
\hline & & & & & \\
\hline & No & $25.2-101.3$ & $60.22 \pm 26.44$ & & \\
\hline
\end{tabular}

Significant $(P<0.05)$ 


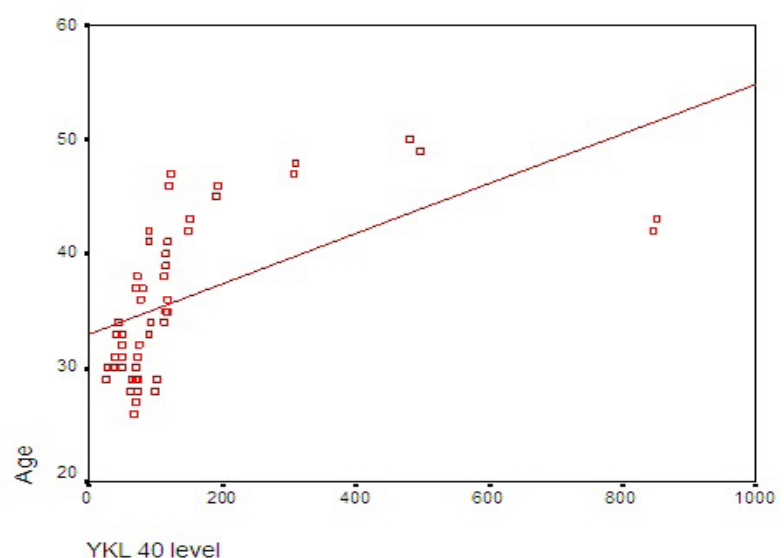

Fig. 1: The correlation between age and serum YKL-40 levels in patients.

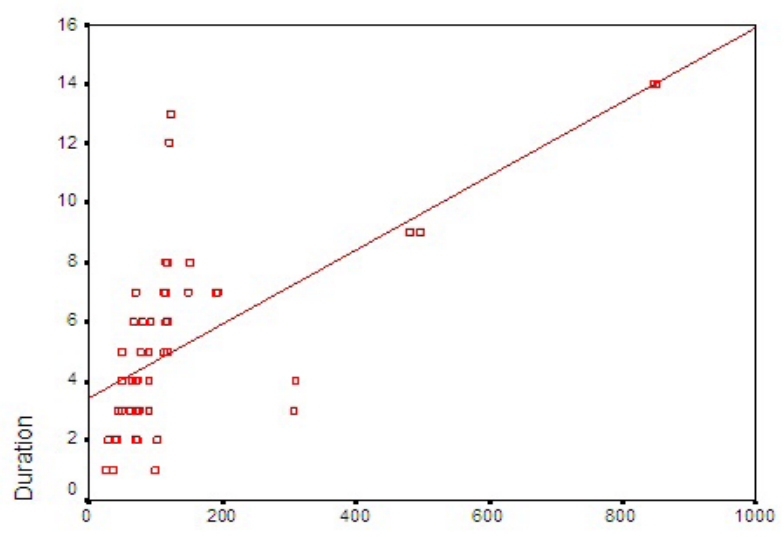

YKL 40 level

Fig. 2: The correlation between duration of erectile dysfunction and serum YKL-40 levels in patients.

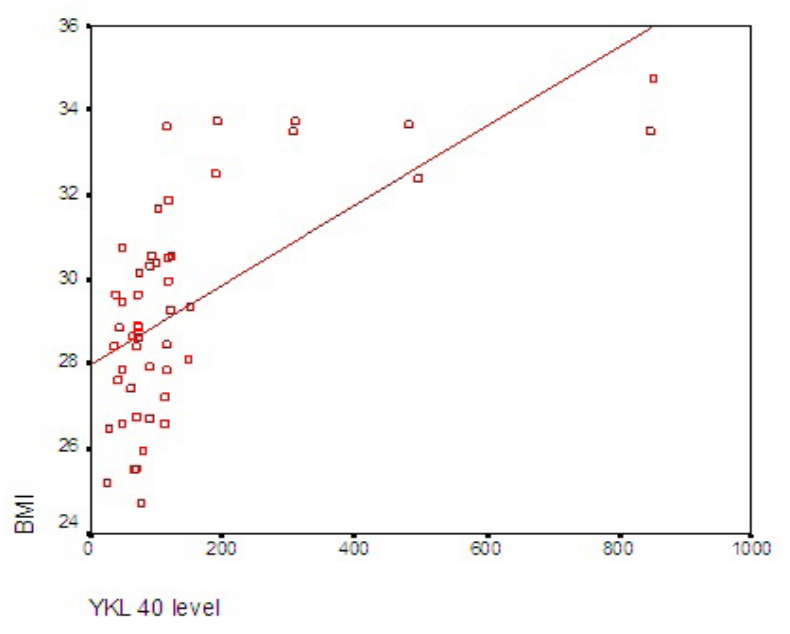

Fig. 3: The correlation between BMI and serum YKL-40 levels in patients.

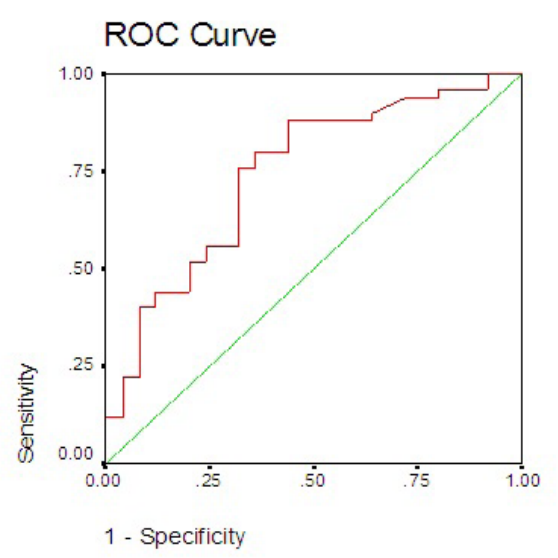

Fig. 4: ROC curve of YKL- 40 levels.

\section{DISCUSSION}

YKL-40 is a marker of inflammation and endothelial dysfunction. It is a growth factor for several cell types and has a known role in extra cellular matrix remodeling and angiogenesis. YKL-40 plays an important role in processes during the early stages of atherosclerosis; moreover, it seems to be of pathogenic importance in the low-grade inflammation that precedes the development of cardiovascular disease $\mathrm{e}^{[8]}$.

Sexual dysfunction is considered to be of vascular origin in the majority of the patients, due to atherosclerotic lesions of the penile arteries. Therefore, it is thus not surprising that sexual dysfunction is more frequently seen in patients with cardiovascular disease with risk factors than in individuals without such conditions. Epidemiological data indicate that sexual dysfunction is frequently found in hypertensive patients and its prevalence is even higher when other cardiovascular risk factors co-exist $\mathrm{t}^{[9]}$.

This study shows that serum marker YKL 40 is significantly higher in arteriogenic ED patients compared with controls. This study demonstrated for the first time the association between serum markerYKL40 as an early marker of endothelial dysfunction with arteriogenic erectile dysfunction.

Behr-Roussel et al. ${ }^{[10]}$, reported that men with early atherosclerosis and coronary endothelial dysfunction have increased prevalence of ED compared with men with normal coronary endothelial function.

Endothelial dysfunction is found in patients with ED, particularly in the early phase of the disease. In a later phase, other factors, such as decreased arterial flow of hypogastric/ pundendal arteries, cavernosal fibrosis, and hypoxia, cause and maintain sexual dysfunction ${ }^{[11,12]}$. 
that vascular endothelial dysfunction develops with increased age in humans in the absence of clinical CVD and major risk factors for CVD. Reduced fibrinolytic function, increased leucocyte adhesion and/or other markers of endothelial dysfunction have been observed in older compared with young adult humans.

Increasing age has been considered as one of the main factors that predisposes people to endothelial dysfunction. The mechanism of endothelial dysfunction during the aging process is accompanied by inbalance between a reduction of NO bioavailability and an increase in the production of cyclooxygenase-derived vasoconstrictor factors. It has been reported that there is a reduced expression and activity of eNOS as well as a decreased expression of $\mathrm{NO}$ and its activity. While endothelium-derived contracting factors, such as ET-1 and cyclooxygenase-derived prostanoids, and ROS production are increased. Plasma levels of ADMA are also known to rise with increased age ${ }^{[23]}$.

Furthermore, in this study, a positive significant correlation was found between serum level of YKL40 and duration of erectile dysfunction, indicating more pathological changes in endothelium occurring with the increase in the duration of ED. ED and vascular diseases share a similar pathogenic involvement of NO pathway leading to impairment of endotheliumdependent vasodilatation (early phase) and structural vascular abnormalities (late phase). ED may be considered to be the clinical manifestation of penile circulation disease that is frequently part of major vascular diseases and that may be an early marker of subclinical atherosclerosis ${ }^{[4]}$.

The results of the present study indicate that YKL40 was positively correlated with BMI of patients. Obesity is a major risk factor for the metabolic syndrome, vascular disease, diabetes, hypertension, endothelial dysfunction and androgen deficiency, all of which have a role in the pathophysiology of erectile dysfunction. Approximately $25 \%$ of obese individuals have metabolic syndrome and obesity, as manifested by an increased body mass index, waist circumference and waist-to-hip ratio, which are responsibe of increased prevalence of $\mathrm{ED}^{[24]}$.Visceral adipose tissue secretes a host of biochemical modulators and proinflammatory factors contributing to systemic and peripheral vascular inflammation. These include interleukin (IL)-6, IL-1b, plasminogen activator inhibitor-1 (PAI-1), tumor necrosis factor- $\alpha$ (TNF $\alpha)$, angiotensinogen, angiotensin-converting enzyme, vascular endothelial growth factor, and serum amyloid A. Adipokines are considered to facilitate monocyte adhesion and migration into the vascular wall and the conversion of monoctyes to macrophages ${ }^{[25]}$. Increased levels of TNF $\alpha$ cause the enhanced expression of
The role of the penile endothelium in the erection process is by producing nitric oxide (NO), which is responsible for the dilation of the penile artery, relaxation of the corpus cavernosa and consequently erection. Thus, dysfunction of the penile endothelium, as found in some disorders, might play an important role in the pathophysiology of $\mathrm{ED}^{[13]}$.

Endothelial dysfunction has been related to most risk factors for atherosclerosis, such as diabetes, dyslipidemia, hypertension, smoking, aging, and obesity $^{[14]}$.

The presence of many risk factors, each playing a role in the development of impaired $\mathrm{NO}$ bioavailability by different mechanism. Accordingly, endothelial function in the coronary circulation was found to be inversely associated with the number of risk factors ${ }^{[15]}$

The present study shows that $39(78 \%)$ arteriogenic ED patients are hypertensive which has an important role in pathophysiology of ED, this was in accordance with other studies ${ }^{[16,17]}$.

The patient's risk-factor profile is not the only determinant of endothelial dysfunction. Low-grade subclinical inflammation has been identified as an additional factor affecting endothelial function in all stages of the atherosclerotic process ${ }^{[18]}$.

In the present study, the mean serum levels of YKL- 40 in hypertensive ED patients was significantly higher $(P<0.05)$ than that measured in serum samples of non-hypertensive patients.

As regards HTN, as another risk factor of endothelial dysfunction, Li et al. ${ }^{[19]}$ and Munzel et al. ${ }^{[20]}$, found that hypertension-related endothelial dysfunction has been found to be the result of increased production of ROS or increased nitric oxide breakdown. In hypertensive patients, endothelial function has been related to target organ vascular damage.

Data supporting the clinical significance of endothelial dysfunction came from the associations with microvascular and macrovascular alterations, and there by with target organ damage, early in the course of essential hypertension. This suggests that endothelial dysfunction, that precedes microvascular and macrovascular alterations, may be used as an early diagnostic marker of preclinical target organ damage both in high-risk populations in which vascular damage is already established and in low-risk individuals who will benefit most by more aggressive treatment ${ }^{[21]}$.

In the present study, we demonstrated a positive significant correlation between serum levels of YKL40 and age of patients. Ungvari et al. ${ }^{[22]}$, showed 
adhesion molecules in both the endothelium and in vascular smooth muscle cells, and IL-6 stimulates liver production of $\mathrm{C}$-reactive protein, a nonspecific marker of vascular inflammation. Another mechanism was suggested, a mechanism whereby TNF $\alpha$ released from fat stores surrounding a vessel may contribute to the dysregulation of insulin modulation of endothelin-1 mediated vasoconstriction and NOmediated vasodilatation favoring vasoconstriction ${ }^{[26]}$.

\section{CONCLUSION}

In conclusion, serum levels of YKL-40 are elevated in arteriogenic ED patients. It could be a novel marker of endothelial dysfunction especially in hypertensive patients. The results need to be further verified by other studies.

\section{CONFLICT OF INTEREST}

There are no conflicts of interest.

\section{REFERENCES}

1. McCabe M and Matic H. Severity of ED: relationship to treatment-seeking and satisfaction with treatment using PDE5 inhibitors. J Sex Med. 2007; 4(1):145-151.

2. Andersen I, Heitmann BL, and Wagner G. Obesity and sexual dysfunction in younger Danish men. J Sex Med.2008; 5:2053 -2060.

3. Chiurlia E, D'Amico R, Ratti C, Granata AR, and Romagnoli R.. Subclinical coronary artery atherosclerosis in patients with erectile dysfunction, $\mathrm{J}$ Am Coll Cardiol. 2005; 46:1503- 1506.

4. Gazzaruso C, Solerte SB, Pujia A et al. Erectile dysfunction as a predictor of cardiovascular events and death in diabetic patients with angiographically proven asymptomatic coronary artery disease: a potential protective role for statins and 5-phosphodiesterase inhibitors. J Am Coll Cardiol.2008; 51: 2040-2044.

5. Jefri M, Huang YN, Huang WC, Tai CS, and Chen WL. YKL-40 regulated epithelial-mesenchymal transition and migration/invasion enhancement in non-small cell lung cancer. BMC. Cancer 2015; 15:15921593-

6. Astrup AS, Tarnow L, Pietraszek L, Schalkwijk CG, Stehouwer CD. Parving HH, and Rossing P. Markers of endothelial dysfunction and inflammation in type 1 diabetic patients with or without diabetic nephropathy followed for 10 years. Diabetes Care.2008; 31:1170-1176.
7. Ober C, Tan Z, Sun Y et al. Effect of variation in CHI3L1 on serum YKL-40 level, risk of asthma, and lung function. New Eng. J. Med. 358: 1682-1691, 2008.

8. Rathcke $\mathrm{CN}$ and Vestergaard H. YKL-40--an emerging biomarker in cardiovascular disease and diabetes. Cardio vasc Diabetol.2009; 8: 61.

9. Mancia G, De Backer G, Dominiczak A, et al. Guidelines for the Management of Arterial Hypertension: The Task Force for the Management of Arterial Hypertension of the European Society of Hypertension (ESH) and of the European Society of Cardiology (ESC). J Hypertens.2007; 25:1105-1187.

10. Behr-Roussel D, Chamiot-Clerc P, Bernabe J, Mevel K, Alexandre L, Safar ME. and Giuliano F. Erectile dysfunction in spontaneously hypertensive rats: pathophysiological mechanisms. Am J Physiol Regul Integr Comp Physiol.2003; 284:R682-R688.

11. Bocchio M, Desideri G, Scarpelli P, et al. Endothelial cell activation in men with erectile dysfunction without cardiovascular risk factors and overt vascular damage. J Urol.2004; 171:1601-1604.

12. Vlachopoulos C, Rokkas K, Ioakeimidis N and Stefanadis C. Inflammation, metabolic syndrome, erectile dysfunction and coronary artery disease. Eur Urol.2007; 52:1590-600.

13. 13- Giuliano F and Droupy S. Erectile dysfunction. ProgUrol.2013; 23 (9): 62937-.

14. 14- Mudau M, Genis A, Lochner A and Strijdom H. Endothelial dysfunction: the early predictor of atherosclerosis. Cardiovasc J Afr.2012; 23:222-231.

15. Brunner H, Cockcroft JR, Deanfield J, et al. Endothelial function and dysfunction. Part II: Association with cardiovascular risk factors and diseases: a statement by the Working Group on Endothelins and Endothelial Factors of the European Society of Hypertension. J Hypertens. 2005; 23:233-246.

16. Nunes KP, Labazi $\mathrm{H}$ and Webb RC. New insights into hypertension-associated erectile dysfunction .Curr Opin Nephrol Hypertens.2012; 21: 163- 170.

17. Idung AU, Abasiubong F, Ukott IA, Udoh SB and Unadike BC. Prevalence and risk factors of erectile dysfunction in Niger delta region, Nigeria. Afr Health Sci.2012; 12: 160- 165.

18. Montorsi P, Ravagnani PM, Galli S, et al. Association between erectile dysfunction and coronary artery disease. Role of coronary clinical presentation and 
extent of coronary vessels involvement. Eur Heart J.2006; 27:2632-2639.

19. Li Q, Youn JY and Cai H. Mechanisms and consequences of endothelial nitric oxide synthase dysfunction in hypertension. J Hypertens.2015; 33:1128-1136.

20. Munzel T, Gori T, Bruno, RM and Taddei S. Is oxidative stress a therapeutic target in cardiovascular disease? Eur Heart J.2010; 31:2741-2748.

21. Rochette L, Lorin J, Zeller $\mathrm{M}$, et al. Nitric oxide synthase inhibition and oxidative stress in cardiovascular diseases: possible therapeutic targets? Pharmacol Ther.2013; 140(3):239-257.

22. Ungvari Z, Kaley G, de Cabo R, Sonntag WE and Csiszar A. Mechanisms of Vascular Aging: New Perspectives. J Gerontol A Biol Sci Med Sci.2010; (10): 1028-1041.
23. Herrera MD, Mingorance C, Rodriguez-Rodriguez R and Alvarez de Sotomayor M. Endothelial dysfunction and aging: an update. Ageing Res Rev.2010; 9:142-152.

24. Nguyen NT, Magno CP, Lane KT, Hinojosa MW and Lane JS. Association of hypertension, diabetes, dyslipidemia, and metabolic syndrome with obesity findings. J Am Coll Surg.2008; 207:928-934.

25. Phillips LK and Prins JB. The link between abdominal obesity and the metabolic syndrome. Curr Hypertens Rep.2008; 10, 156-164.

26. Ridker PM, Danielson E, Fonseca FA et al Rosuvastatin to prevent vascular events in men and women with elevated C-reactive protein. N Engl J Med.2008; 359: 2195-2207. 